\title{
Der "Aufbewahrungsort des Falschen" - Fehler und Zuä̈lle in Wolfgang Herrndorfs Roman Sand am Beispiel des Homonyms Mine
}

[The place of preservation of the false - mistakes, accidents and homonyms in the novel Sand by Wolfgang Herrndorf]

Sonja Arnold ${ }^{1}$

\begin{abstract}
This paper aims to examine Wolfgang Herrndorf's novel Sand (2011), taking into consideration its concatenation of errors, accidents and the logic of the absurd. The first part of this investigation regards the different connotations of the desert motif and their associated traditions. Secondly, it will be shown how these thematic expressions of the anti-logic can be applied to the level of reception, regarding formal categories, such as narrative position, time structure and plot patterns. Finally, with the example of the homonym Mine and its homophone Miene the paper will demonstrate how the novel plays with (semantic) misunderstandings and misinterpretations, becoming a "repository of the false."
\end{abstract}

Keywords: Contemporary literature - Wolfgang Herrndorf - metaphor of the desert homonyms

Zusammenfassung: Der vorliegende Beitrag untersucht Wolfgang Herrndorfs Roman Sand (2011) auf die ihm zu Grunde liegenden Verkettungen von Fehlern, Zufällen und die Logik des Absurden. Es werden dabei zunächst die verschiedenen Konnotationen des Wüstenmotivs und ihre dazugehörigen Traditionslinien in den Blick genommen. Im Anschluss wird gezeigt, inwiefern diese thematische Ausprägung des Antilogischen auch auf die Ebene der Rezeption übertragen werden kann, indem formale Kategorien wie Erzählposition, Zeitstruktur und Plotmuster untersucht werden. Am Beispiel des Homonyms Mine und seines Homophons Miene wird schließlich herausgearbeitet, wie der Roman mit (semantischen) Missverständnissen und Fehlinterpretationen spielt und solchermaßen zum „Aufbewahrungsort des Falschen“ wird.

Stichwörter: Gegenwartsliteratur - Wolfgang Herrndorf - Wüstenmetapher - Homonyme

1 Professora visitante do DAAD na Universidade Federal do Rio Grande do Sul. Email: daad_porto_alegre@daad.org.br 


\section{Einleitung}

„In the desert, you can remember your name“, heißt es in einem bekannten Lied der Gruppe America aus dem Jahr 1972, ${ }^{2}$ das damit auf die romantische Vorstellung der Selbstfindung auf einer Reise in die Fremde $^{3}$ rekurriert, in der gerade die äußere Kargheit die innere Mannigfaltigkeit erblühen lässt. Den Gegenentwurf hierzu bildet die Einsicht, dass Identität und Selbsterkenntnis nur in der Interaktion mit anderen gefunden werden können - eine Einsicht, die sich von der dialogischen Philosophie (BUBER 1962) über die Neurobiologie ${ }^{4}$ bis zur Literatur ${ }^{5}$ durch eine Vielzahl von Disziplinen zieht. Dieser Linie folgend, ruft die Wüstenkonnotation in Wolfgang Herrndorfs Roman Sand (2011) in Form eines Selbst-, Namens- und Gedächtnisverlusts eine gegenteilige Vorstellung auf - diejenige der Wüste als Ort des Fremden, Anderen und Bedrohlichen. ${ }^{6}$ Dafür spricht auch, dass mehreren Kapiteln des Romans in Form von Motti Zitate des antiken Geschichtsschreibers Herodot voranstehen, der mit seinen Beschreibungen der Wüste die Tradition des Anderen ${ }^{7}$ und Bedrohlichen einleitete (vgl. LindEMANN/SCHMITZ-EMANS 2000: 10). Ganz im Gegenteil zur Selbstfindung bleibt die in der Wüsteneinsamkeit gewonnene Erkenntnis eine vermeintliche. Immer wieder werden falsche Fährten gelegt, Theoriegebäude konstruiert und wieder verworfen, die oftmals nur haarscharf an der tatsächlichen Lösung vorbeigehen. Diese ist indes freilich ebensowenig in allen Fällen zweifelsfrei auszumachen. Aber gerade im Modus des Knapp daneben wird im Roman ein Netz von Fehlschaltungen, fatalen Irrtümern und unheilvollen Zufallsverkettungen um den Protagonisten mit Gedächtnisverlust

2 America: A horse with no name (1972).

3 Vgl. etwa TIECKS Franz Sternbalds Wanderungen (1798) oder Joseph von EICHENDORFFS Aus dem Leben eines Taugenichts (1822/23).

4 Vgl. beispielsweise Harald WELZERS (2002) Studien zum kommunikativen Gedächtnis oder Eric KANDELS (2009) Grundlagenwerk Auf der Suche nach dem Gedächtnis.

5 Vgl. beispielsweise Paul GoETSCHS (2000) Studie über Robinson Crusoe.

6 Vgl. „Meist stand sie [die Wüste] für Werte, die denen der Stadt entgegengesetzt waren.“ (LINDEMANN/SCHMITZ-EMANS 2000: 9).

7 Vgl. beispielsweise das dem ersten Kapitel voranstehende Zitat Herodots, das auf die Andersartigkeit der Bewohner Afrikas abzielt: „Wir schicken jedes Jahr - und scheuen dabei weder Leben noch Geld ein Schiff nach Afrika, um Antwort auf die Fragen zu finden: Wer seid ihr? Sie aber schicken nie ein Schiff zu uns“ (HERRNDORF 2011: 7). 
gesponnen. Herrndorf selbst bezeichnet den Roman dabei in seinem Blog $\underline{\text { Arbeit und }}$ Struktur als „Aufbewahrungsort des Falschen.“ 8

Diese Logik des Irrtums lässt sich im Roman auf mehreren Ebenen festmachen, die im Folgenden genauer in den Blick genommen werden: Motivisch lassen sich im Roman beide zuvor bereits skizzierten Traditionslinien des Wüstenbegriffs nachzeichnen. Formalästhetisch weist der Roman eine Vielzahl von Erzählpositionen auf: auktoriale, personale sowie eine Ich-Rahmen-Erzählung, die jedoch nur an zwei Stellen des Textes erkenntlich wird. Schließlich wird der Gedächtnisverlust auch auf der Ebene der histoire zum zentralen Thema. Die logischen Fehlschlüsse, die sich zunächst im Modus der Rezeption auf der formalen Ebene ergeben, lassen sich auch innerhalb der intradiegetischen Welt nachvollziehen: zunächst aufgrund eines Protagonisten mit defizitären Hirnfunktionen und schließlich aufgrund semantischer Ähnlichkeitsspiele mit Homonymen, Homographen und Homophonen.

Die Grundlage hierfür bildet Wolfgang Herrndorfs Roman Sand (2011), der 2012 mit dem renommierten Preis der Leipziger Buchmesse ausgezeichnet wurde. Im Jahr 1972 geschehen in einem nordafrikanischen Land merkwürdige Dinge, die alle um einen Mord in einer Wüstenkommune angeordnet sind. Der vermeintliche Täter, Amadou Amadou, wird von den Kommissaren Canisades und Polidorio ins Visier genommen. Letzerer verschwindet nach dem ersten Teil des Romans spurlos und erwacht im zweiten Teil nach einem Schlag auf den Kopf ohne Gedächtnis in einer Scheune. Fälschlicherweise wird er von der CIA-Agentin Helen Gliese für den Drahtzieher einer bedrohlichen Aktion gegen die westliche Welt gehalten (der vermutlich tatsächlich darin verstrickte Agent Lundgren ist zu diesem Zeitpunkt schon tot) und es beginnt für ihn eine rastlose Flucht und eine Jagd nach der Wahrheit, die jedoch immer wieder in Missverständnisse und nicht zu klärenden Theorien mündet. Eine wesentliche Rolle spielt hierbei die Raumsemantik, die in Form einer Düne auf dem Buchumschlag ${ }^{9}$ Assoziationen einer undurchschaubaren, unabschließbaren und fremden Landschaft hervorruft. Dementsprechend bleiben einige Geschehnisse und logische Zusammenhänge sowohl für die Figuren der intradiegetischen Welt als auch für die Rezipienten unentschlüsselbar. Die Wüstenkonnotation zeigt sich mithin sowohl

8 http://www.wolfgang-herrndorf.de/2011/09/zwanzig/ (31/01/2013).

9 Vgl. zu dieser Traditionslinie auch Bodo KIRCHHOFFs (1992) Roman Der Sandmann und dessen Umschlaggestaltung. 
Arnold, S. - Der "Aufbewahrungsort des Falschen"

auf der formalen Ebene durch eine Vielzahl von unterschiedlichen Erzählpositionen und dem Spiel mit verschiedenen Erzählmustern als auch im Versuch einer semantischen Entschlüsselung, die sich um das Wort Mi(e)ne gruppiert.

\section{Die Wüste als Ort des Absurden}

Wüste, Sand und Sandmann haben eine lange Tradition in der Literatur- und Kulturgeschichte. Meist sind diese Motive dabei mit dem Phänomen des Transitorischen verbunden - zwischen Träumen und Wachen sowie zwischen Realität und Fiktion, so etwa in KIRCHHOFFS Roman Der Sandmann (1992) oder in E.T.A. HofFMANNS Novelle Der Sandmann (1816), in der die Realitätswahrnehmungen des Protagonisten Nathanael schwanken. Die Wüste kann aber auch der Ort des Anderen, des Bösen (vgl. hierzu auch den Arbeitstitel des Romans Die Wüsten des Bösen, vgl. MAAR 2012: 340) sowie des radikal Gleichgültigen sein. Herrndorfs Roman vereint diese Vorstellungen und ruft unterschiedliche Konnotationen des Wüstenbegriffs auf, die eng mit den thematischen Verstrickungen des Romans verbunden sind.

Nachdem der gedächtnislose Protagonist, der sich nach dem Label seines Anzugs Carl nennt, das Geheimnis um die Mine entschlüsselt zu haben glaubt und ihm mit zwei in einer Mine versteckten Kapseln die Flucht in die Wüste gelingt, liegt er auf einer Wüstendüne und reflektiert seine Einsamkeit. Bereits zu Beginn dieses Kapitels wird auf die Vorstellung eines zunehmenden Selbstverlusts Bezug genommen und es heißt:

Weiß einer, wie es ist, die Nacht in der Wüste zu erleben, allein? Wer gewohnt ist, seine Nächte in einem Bett zu verbringen, in einem Haus, umgeben von anderen Häusern und Menschen, macht sich davon nur schwer eine Vorstellung. Und noch schwerer macht man sich eine Vorstellung davon, wie die Schwärze und Finsternis der Metaphysik an einem Geiste zerren kann, der in sich selbst seit Tagen nichts weiter zu erkennen vermag als ein unbeschriebenes Blatt Papier.

Als Gegensatzbegriff zur Zivilisation wird oft Barbarei genannt, doch ein passenderes Wort wäre im Grunde Einsamkeit. (HERRNDORF 2011: 308). 
Die im Popsong postulierte verstärkte Erinnerungsleistung aufgrund einer Situation, die frei von äußeren Einflüssen ist, wird hier in ihr Gegenteil verkehrt. Die unendliche Stille und unabschließbare Dunkelheit der Wüste werden zum Mysterium und für den Protagonisten bleiben lediglich „Schwärze und Finsternis“; er selbst wird im Sinne der Abwesenheit identitätskonstituierender Erinnerungen zum „unbeschrieben[en] Blatt“. War er in der Interaktion mit Helen und seinen Verfolgern zumindest auf der Suche nach seiner Identität, so vermag ihm die Wüste nichts zurückzugeben, sie bleibt der Ort des Fremden, absolut Einsamen, der am Ende des Zitats in einen Gegensatz zur Zivilisiation, mithin der Idee einer Identitätsformation durch Interaktion und Kommunikation gesetzt wird. Das Wüstenmotiv wird hierbei mit dem Motiv der Nacht kombiniert, das auf das Defizitäre und Undurchsichtige hinweist und am Ende des Romans im vergeblichen Versuch, die Dunkelheit der Höhle zu verlassen und endlich die Identität wiederzufinden, erneut erscheint (vgl. HERRNDORF 2011: 433). Das Fehlen eines Gegenübers wird zudem im Motto dieses Kapitels durch das Gedicht To the Moon (HERRNDORF 2011: 308) des britischen Romantikdichters Percy B. Shelley aufgenommen, das die rastlose und einsame Wanderung des Mondes beschreibt.

Indes herrschen im Roman auch andere Vorstellungen der Wüste vor. Im Sinne des eingangs zitierten Popsongs der Gruppe America wird in der Kommune, in der der Vierfachmord stattgefunden hat, gerade die Idee einer Selbstfindung und positiven Veränderung unter Einwirkung der Wüstenlandschaft propagiert - schließlich war diese Wirkung ein wesentlicher Ansatzpunkt für die Wahl des Standorts der Kommune, die ausschließlich von Ausländern bewohnt wird. Im Gespräch zwischen Helen und dem geistigen Führer der Kommune, Fowler, erklärt Letzterer die Veränderungen der Jugendfreundin Michelle mit der besonderen Umgebung: „Die Wüste verändert dich. Der Nomade. Wenn einer lange hier gelebt hat, wird sein Blick ein anderer. Der Wüstenbewohner ist ruhiger. Er ist das Zentrum. Er geht nicht auf die Dinge zu, die Dinge gehen auf ihn zu.“ (HERRNDORF 2011: 121). Dieses Credo der Kommunenbewohner wird indes dadurch unterlaufen, dass in der Sicht Helens überhaupt keine wesentliche Veränderung stattgefunden hat: Michelle wird von ihr weiterhin als ein schwacher, orientierungsloser und naiver Mensch wahrgenommen.

Zwischen slapstickartigen Elementen und grotesken Ausformungen, die schon Herrndorfs Roman Tschick (2010) bestimmten, und tragischen Verwicklungen, ist die 
Grundstimmung des Romans schwer auszumachen. Sie kann jedoch, wie es Michael Maar beschreibt, treffend mit dem Begriff des Absurden charakterisiert werden: „Das zutiefst Grausige berührt sich mit dem Komischen in einem Punkt: dem Absurden.” (MAAR 2012: 339). Liest man die Wüstenlandschaft vor diesem Hintergrund, so wird ein weiterer Bezugsrahmen eröffnet: die Beschreibungen der maghrebinischen Wüste in Albert CAMus' (1994) Roman Der Fremde. Es sind gerade die ungeheure Leere der Wüste, ihre End- und Sinnlosigkeit sowie die dumpfe Gewalt der stechenden Sonne, die den Protagonisten aus Camus' Roman zum Mörder ohne Moral und Gewissen werden lassen. Die daraus folgende Philosophie des Absurden liegt Herrndorfs Roman nicht nur durch die strukturelle Verknüpfung willkürlicher Zufälle zu Grunde, sie wird zur expliziten Camus-Referenz, indem im Gespräch mit dem Psychiater Dr. Cockcroft nach „Sisyphus“ (HERRNDORF 2011: 197) gefragt wird (eine Referenz auf CAMUS' (1999) zentrales Werk Der Mythos des Sisyphus, in dem er die Philosophie des Absurden wesentlich entwickelt) und die Wüstenreferenz explizit an die Gedanken über die Sinnhaftigkeit des Daseins und dessen logische Folge geknüpft wird: „der Gedanke drängte sich auf, dass unter den Bedingungen dieser Landschaft nicht nur ein Menschenleben unbedeutend war, sondern, philosophisch gesprochen, auch vier Menschenleben oder das Leben der ganzen Menschheit.“ (HERRNDORF 2011: 76). Dieser Gedanke kommt Polidorio, als er nach einer Verfolgungsjagd durch die Stadt Targat in der Sandwüste angekommen auf einer Düne die Vorboten eines Sandsturms erblickt. Unmittelbar verknüpft ist dieser Gedankengang mit der Erinnerung an die absurden Ereignisse um den Vierfachmord in der Kommune und den vermeintlichen Täter Amadou Amadou. Er fungiert damit gleichsam als Vorbote der kommenden Ereignisse, die alle unter dem Schild des radikalen Zufalls stehen und die absurde Verkettung unglücklicher Umstände im Leben Polidorios alias Carl alias Cetrois vorwegnehmen. Die Unbedeutsamkeit der menschlichen Schicksale zeigt sich dann auch in der Schlussszene des Romans. Das kleine Mädchen, das am Ende der Kette in den Besitz der Mine gelangt und diese als Stütze für seine Puppe benutzt, wird gemeinsam mit der gesamten Wohnsiedlung für immer verschüttet. Entscheidend an dieser Stelle ist, dass Polidorio die Bedeutungslosigkeit eines Menschenlebens an die „Bedingungen dieser Landschaft“" koppelt, wie es schon Camus' Held Meursault in Der Fremde vornimmt. Auch andere Figuren des Romans teilen diese Einschätzung und leiden unter der Gewalt der Hitze und der Orientierungslosigkeit in der 
Arnold, S. - Der "Aufbewahrungsort des Falschen"

Wüstenlandschaft. Der schwedische Agent Lundgren wird solchermaßen immer wieder mit der Klage „und die Sonne“ (HERrNDORF 2011: 65) zitiert. Die Sonne hat hierbei auch zahlreiche Nebenwirkungen auf Lundgrens Sprachzentrum, eine Vorausdeutung auf die Sprachspiele und das angeschlagene Denkvermögen Carls im Fortgang des Romans. So ist es die Sonne, die dazu führt, dass bisher klar umrissene Zusammenhänge, Benennungen und logische Folgen verschwinden: „die elektromagnetische Strahlung brannte durch den Strohhut, und plötzlich war das Wort weg." (HERRNDORF 2011: 65). Bereits hier etabliert sich in der semantischen Dimension die Logik des Knapp daneben, die sich mithin nicht nur auf Polidorio/Carl bezieht. Im Folgenden werden in einer Prolepse bereits die zentralen Elemente des tip of the tonguePhänomens, ${ }^{10}$ des Wörterbuchs und der Homophone als zentrale Themen des Sprachverlusts Polidorios vorweggenommen, die an dieser Stelle ursächlich auf die Wüstenlandschaft zurückzuführen sind:

Ein taubes Gefühl blieb wie Watte auf seiner Zunge zurück. Das Wort war weg. Es war, als würde er sich nicht an seinen eigenen Namen erinnern. Er erinnerte sich nicht an den Namen vom Dings. Das Dings, das sich drehte. Weswegen er hier war. Klar, Zentrifuge, zentrifugal. Das kam gleich neben Zentaur, Zentrum, Zentralgestirn. Die Zentrifuge, sicher. Und davor? Es wurde immer schlimmer. Vorhin hatte er schon Münztee gedacht. Mademoiselle, ein Münztee. (Herrndorf 2011: 65).

In der Folge werden in personaler Erzählperspektive verfremdete Phraseologismen wiedergegeben, die ebenso der Logik des Knapp daneben folgen. So „zitterte Lundgren wie Erbsenlaub“ (HERRNDORF 2011: 66) anstelle von „Espenlaub“. Die Serviererin im Café war „Dumm wie Binsenstroh“ (HERRNDORF 2011: 67) anstelle von Bohnenstroh. Die genuine Eigenschaft der Phraseologismen, feste, unveränderliche Wortverbindungen zu formen (KAISER 2007: 585), wird hier unterlaufen und deutet gleichzeitig auf die verschobene Phonetik im weiteren Verlauf des Romans voraus. Carl verwechselt Laute aufgrund eines Schlags auf den Kopf, mithin aufgrund einer funktionalen Störung im Gehirn, für Lundgrens Verwechslungen zeigen sich Sonne und Wüstenklima verantwortlich, die damit sowohl Sprechfertigkeit als auch logisches

10 Ein Phänomen der Aphasiologie, bei dem auf ein Wort nicht mehr zugegriffen werden kann, den Betroffenen jedoch die Anfangslaute auf der Zunge liegen. Vgl. auch Carls Schrei in der Höhle am Ende des Romans, wenn er endlich Zugriff auf den Namen hat, „der ihm schon die ganze Zeit auf der Zunge gelegen hatte" (HERRNDORF 2011: 444). 
Arnold, S. - Der "Aufbewahrungsort des Falschen"

Denken entscheidend beeinträchtigen. In diesem Sinne bezieht sich auch das Motto dieses Kapitels auf ein logisches Paradoxon („,When I hear of Schrödinger's cat, I reach for my gun. Stephen Hawking“, HERRNDORF 2011: 62). Bei Schrödingers Katze handelt es sich um ein Gedankenexperiment der Quantenmechanik, das den Zustand eines Atomkernzerfalls beschreibt, bei dem nach der Theorie der Quantenmechanik ein Zeitpunkt existiert, zu dem ein Atomkern zerfallen und nicht zerfallen ist. Demnach müsste eine sich in diesem Raum befindende Katze zu einem bestimmten Zeitpunkt gleichzeitig lebendig und tot sein. Dieses logische Paradoxon korrespondiert mit den logischen Fehlschlüssen sowohl dieses Kapitels als auch des gesamten Romans.

Die Unfähigkeit, unter diesen Eindrücken Sinn zu generieren, lässt sich auch auf der Handlungsebene aufzeigen, die unter denselben Einflüssen eine von absurden Zufällen vorangetriebene ist. ${ }^{11}$ So lässt sich schon zu Beginn des Romans über Polidorios Berufswahl proleptisch lesen: „Wie so vieles in seinem Leben war es Zufall““ (HERRNDORF 2011: 17). Die Kette von falschen Schlussfolgerungen in einer Logik des Knapp daneben zieht sich durch den gesamten Roman und fungiert als Handlungsmotor. So spricht der auktoriale Erzähler am Ende des Romans von Carl als „eine[m] Mann, der weder willentlich noch zufällig unter die Räder des Schicksals geriet, sondern einzig und allein durch eine falsche logische Schlussfolgerung; durch den Glauben an die Unschuld eines Schuldigen“" (HERRNDORF 2011: 452). Diese falschen logischen Schlüsse lassen sich indes nicht nur innerhalb des Romans auf der Figuren- und Inhaltsebene nachzeichnen, sie können mithilfe einer Untersuchung von Erzähl- und Plotstruktur auch auf die Ebene der Rezeption ausgeweitet werden.

\section{Formale Kriterien: das Spiel mit Erzählposition, Metafiktionalität und Plotmustern}

Formal sind mit der durch Zufälle vorangetriebenen und vom Absurden gekennzeichneten Handlung eine Vielzahl von Erzählpositionen und Manipulationen der Zeitstruktur verbunden, die eine lineare Entschlüsselung durch den Rezipienten

11 Vgl. die Rezension von Andrea Hanna HüNNIGER in Die Zeit (22.11.2011): „In diesem Werk wird die Handlung fast ausschließlich von Fehlern vorangetrieben.“ http://www.zeit.de/2011/47/L-B-Herrndorf $(31 / 01 / 2013)$. 
zunehmend erschweren. So verwundert es nicht, dass ein großer Teil der Rezensionen zum Roman die Verwirrung und Unentschlüsselbarkeit der histoire in den Mittelpunkt stellt $^{12}$ und nur die Rezensentin der ZEIT tatsächlich auf im Roman doppelt erscheinende Figuren hinweist. Wie Michael MAAR (2012) indes nachweist, lassen sich die im Roman gelegten Fährten durchaus auflösen und die Identität der einzelnen Personen klar benennen. So zeichnet Maar in seinem Beitrag minutiös die Identität der Figuren Polidorio, Carl und Cetrois nach: denn „Polidorio ist gleich Carl ist gleich Cetrois, die drei scheinbar getrennten Figuren sind ein und dieselbe" (MAAR 2012: 335). Nachdem der Kommissar Polidorio, an die Unschuld Amadou Amadous glaubend, noch einmal in die Wüste fährt, verschwindet er. Gleichzeitig - im Roman jedoch in einem anderen Segment, im zweiten von fünf Büchern mit dem Titel Die Wüste eingeleitet - wacht der namenlose Protagonist mit Gedächtnisverlust niedergeschlagen in einer Scheune auf. Dem aufmerksamen Leser erschließen sich, wie Maar nachweist, zahlreiche Parallelen in der Figurenbeschreibung der beiden. So spricht Carl Arabisch, denkt aber auf Französisch; Polidorio ist in Frankreich aufgewachsen. Carl wundert sich, warum er Deutsch versteht, Polidorio hatte in seiner Kindheit ein Internat in der Schweiz besucht (vgl. HERRNDORF 2011: 216 und MAAR 2012: 335). Schließlich wird er gar von einer Prostituierten und zwei Kommissaren als Polidorio bzw. Cetrois erkannt (vgl. Herrndorf 2012: 170, 322/23). Am Ende werden Polidorios Frau und Kind - der Drohung seiner Widersacher entsprechend - tot aufgefunden, er selbst gilt als verschollen (HERRNDORF 2012: 449, 451). Diese Reihe von Hinweisen ließe sich noch weiter fortsetzen. Herrndorf selbst beklagt das Problem, dass diese Hinweise nicht korrekt entschlüsselt werden, in seinem Blog Arbeit und Struktur:

Viel größeres Problem: Daß die Handlung keiner kapiert. Drei von fünf Lesern konnten den Amnestiker bisher nicht identifizieren, was etwa ist, als verriete ein Krimi den Mörder nicht. Das ist keine Absicht. Riesige Verschwörungstheorien auffahren, Fäden ins Leere laufen lassen und am Ende keine Lösung haben, ist nicht originell, nicht postmodern, sondern einzig und allein ein mächtiger Schmerz im Arsch. ${ }^{13}$

\footnotetext{
12 Vgl. beispielsweise die Besprechungen von Deutschlandradio Kultur: http://www.dradio.de/dkultur/sendungen/kritik/1622678/ (31/01/2013) oder des NDR: http://www.ndr.de/ndr2/programm/sendungen/buchtipp/buchtipp799.html (31/01/2013).

13 http://www.wolfgang-herrndorf.de/page/17/ (31/01/2013).
} 
Wie kommt es also, dass die von Herrndorf vermeintlich so eindeutig angelegten Fäden vom Rezipienten nur partiell verknüpft werden können? Zunächst lässt sich die Verwirrung des Rezipienten bei der Entschlüsselung der Geschichte, die inhaltlich mit der Wüstenmetapher, der semantischen Verwirrung und dem Gedächtnisverlust des Protagonisten verbunden ist, auf die formale Struktur des Romans zurückführen. Der Roman ist aufgeteilt in fünf Bücher: Das Meer, die Wüste, die Berge, die Oase, die Nacht. ${ }^{14}$ Während die Chronologie insofern aufrechterhalten wird, als in den Büchern nacheinander vom Gedächtnisverlust des Protagonisten bis zu seinem Tod erzählt wird, werden bestimmte Handlungsstränge in verschiedenen Büchern an unterschiedlichen Stellen wieder aufgegriffen und erwecken so den Eindruck, sie würden sich zeitlich nacheinander ereignen, während sie doch parallel geschehen, sich ergänzen und vom Leser in eine Chronologie gesetzt werden müssten. So sind der Mord in einer Hippiekommune und die Verstrickung zwischen der dort lebenden Michelle und ihrer Jugendfreundin Helen, die mittlerweile als CIA-Agentin arbeitet, schon im ersten Buch ein Thema und tauchen in den weiteren Büchern, mit anderen Handlungssegmenten verknüpft, wieder auf. Während des ganzen Romans erscheint an unterschiedlichen Stellen auch ein gewisser Lundgren alias Herrlichkoffer, der im siebten Kapitel des 1. Buchs mit den Worten „Und jetzt hatte Lundgren ein Problem. Lundgren war tot.“ (HERRNDORF 2011: 42) eingeführt wurde. Rückblickend wird dann zunächst im selben Kapitel, später in den Kapiteln 10 und 13 von seiner Ankunft, seinem Treffen mit Cetrois und seinem Tod berichtet, während Hintergrundinformationen $\mathrm{zu}$ seiner Herkunft erst in den Schlusskapiteln aufgelöst werden. Dass ein Leser in der Lage ist, die Geschichte in eine chronologische Ordnung zu bringen, die ungleich der narrativen Ordnung ist, „setzt implizit die Existenz einer Art von einem Nullpunkt voraus, wo Erzählung und Geschichte in ihrem zeitlichen Verlauf vollständig koinzidieren würden“ (GENETTE 1998: 23), so der französische Erzähltheoretiker Gérard Genette. Dass dieser Nullpunkt vom Leser nur schwer zu bestimmen ist, hängt auch mit der Zuverlässigkeit der Reflektorfiguren zusammen.

Der Dialog zwischen Lundgren alias Herrlichkoffer und Cetrois alias Polidorio alias Carl beim Zusammentreffen im Café wird am Ende des Romans von einem völlig

14 Diese Motive spielen zudem eine wesentliche Rolle für den Handlungsfortgang. So erstarkt im Buch die Oase, dem Motiv entsprechend, die Hoffnung auf Lösung des Rätsels, jedoch wird diese durch das anschließende und abschließende Buch die Nacht sogleich wieder zertsört. 
dehydrierten und entkräfteten Carl als Fieberphantasie wiedergegeben. Die beiden hatten sich zu Beginn des Romans in einem Straßencafé getroffen, wo Lundgren Polidorio fälschlicherweise für seine Kontaktperson gehalten hatte. Am Ende des Romans wird dieses Zusammentreffen von einem auktorialen Erzähler wiedergegeben und durch gelegentliche Fokalisierungen auf Carl durchbrochen. Dabei wird der Dialog der beiden, ein Segment, das vor Carls Gedächtnisverlust angesiedelt ist und erst im Zuge einiger freigesetzter Erinnerungen ${ }^{15}$ während seines Todeskampfes wieder zum Vorschein kommt, aus dem 10. Kapitel des 1. Buchs, wörtlich wiederholt. Lundgrens Name wird indes im 2. Dialog durch „der Geist“ (HERRNDORF 2011: 455) ersetzt, während „die Handbewegung des anderen“ zu „Carls Handbewegung“ (HERRNDORF 2011: 455) wird. Die scheinbare Fieberphantasie des Protagonisten hat hier also einen ganz klaren Referenzpunkt und bietet durch das Inbezugsetzen zu anderen Stellen des Romans und die Wiederherstellung der Chronologie auf der Ebene der histoire klare Hinweise auf die Entschlüsselung wesentlicher Handlungselemente des Romans, in diesem Fall die Identität des gedächtnislosen Protagonisten mit dem Kommissar Polidorio, der dem Leser zuletzt in der Szene des Zusammentreffens mit Herrlichkoffer begegnet war. Hier wird deutlich mit den Lesegewohnheiten des Rezipienten gespielt, die wesentlich an die Namenskonventionen gebunden sind, mithin von der NichtIdentität anders heißender Charaktere ausgehen und andere erzählerische Elemente, die auf eine Identität hinweisen, dem unterordnen. SCHNEIDER (2000: 38) zufolge sind bei der Informationsvergabe über fiktionale Figuren beim Rezipienten sowohl bottom upals auch top down-Prozesse wirksam. Während erstere den neuen Aufbau einer Information über eine fiktionale Figur meinen, werden bei letzteren bereits gespeicherte Informationen zur neuen Verarbeitung wirksam (vgl. SCHNEIDER 2000: 38). Dementsprechend könnte es an vorliegender Stelle zu einer Revision der These von zwei getrennten Figuren kommen. Dass dies nicht geschieht, kann mit der unterschiedlichen Namensgebung erklärt werden. ${ }^{16}$ Zudem muss in der besprochenen

15 Hierzu gehören beispielsweise die zurückkehrende Erinnerung an das Schlittschuhlaufen mit der Mutter, an New York (HERRNDORF 2011: 439) sowie die Erinnerungen an den Großvater (HERRNDORF 2011: 440).

16 „Figuren werden nicht durch den Text kategorisiert, individualisiert, personalisiert oder entkategorisiert, sondern in durchaus unterschiedlicher Ausprägung durch den Rezipienten“ (SCHNEIDER 2000: 167). Vgl. dazu auch die Definition Eders: „Figuren werden hier als textbasierte Konstrukte des menschlichen Geistes begriffen, zu deren Beschreibung Modelle sowohl des Textverstehens als auch der menschlichen Psyche erforderlich sind“ (EDER 2008: 31). 
Arnold, S. - Der "Aufbewahrungsort des Falschen"

Passage erzähltechnisch Carl als Reflektorfigur gelten. Dabei handelt es sich jedoch um eine Reflektorfigur, die unter Gedächtnisverlust leidet, dehydriert ist und halluziniert. Dies kann in der Folge dazu führen, dass sie vom Rezipienten als nicht zuverlässig eingestuft wird. ${ }^{17}$

Verwirrend für den Leser ist auch die Rahmung durch einen zwischengeschalteten Ich-Erzähler, der im Roman nur an zwei Stellen auftaucht. Zum ersten Mal im 8. Kapitel des 1. Buchs, kurz nachdem ein Taxifahrer das Wort Mine zum ersten Mal völlig unvermittelt verwendet (HERRNDORF 2011: 46 f.): „Meine Eltern hatten ein Zwei-Zimmer-Appartment im neunten Stock gemietet, und wenn sie mich, wie so oft, hinausschickten, um hinter verschlossenen Türen geheimnisvolle Dinge zu treiben, erkundete ich allein das weitläufige Hotelgelände“" (HERRNDORF 2011: 47). Der Erzähler, mittlerweile erwachsen, bewohnt den Ort der Handlung zur Handlungszeit 1972 - und steht auf geheimnisvolle Weise mit Helen in Kontakt, an deren Bungalow er auf dem Weg zum Meer vorbeikommt. Die Fotografie der Tür von Helens Bungalow hängt in der Erzählzeit über seinem Schreibtisch (HERRNDORF 2011: 49). Demnach schaltet sich an dieser Stelle der Schreiber der Geschichte ein und alles bislang Erzählte wird zu einer davon gerahmten Binnenerzählung. Zum zweiten und letzten Mal kommt der Rahmenerzähler am Ende des Romans zum Vorschein, als er einen Brief von Helens Tochter erhält: „In einem Brief schrieb Heather Gliese mir, ihre Mutter habe ein glückliches und erfülltes Leben geführt und sei rüstig, bei guter Gesundheit und wenige Tage vor ihren zweiundsiebzigsten Geburtstag sanft entschlafen“ (HERRNDORF 2011: 451). Hier wird mit der Illusion der Geschichte gebrochen und ein externer Referenzrahmen etabliert: derjenige eines Erzählers der Geschichte, der mit einer realen Helen und deren Tochter bekannt war. Dieser erzähltechnische Bruch entspricht der Verwirrung um Chronologie und Faktizität der Geschehnisse in der Binnenerzählung. Er wird zudem zu einem Spiel mit Fakt und Fiktion ausgeweitet, wenn Heather Gliese in der Danksagung des realen Autors (vgl. hierzu auch MAAR 2012: 338) auftaucht (HERRNDORF 2011: 476) und mit dieser paratextuellen Information klar die Fiktionalitätsebene verlässt. Es handelt sich hierbei um eine explizite Störung der

17 NÜNNING (1998: 27 f.) zufolge sind Unstimmigkeiten und Diskrepanzen in den Erzählsegmenten eines Erzählers ein entscheidendes Kriterium für die Unzuverlässigkeit von Erzählerfiguren. Diese tauchen in der Schlusspassage, in der der dehydrierte und halluzinierende Carl teilweise als Reflektorfigur fungiert, zuhauf auf: ,irgendeine synästhetische Fehlschaltung verwandelte seine Schreie in Farben“ (HERRNDORF 2011: 437). 
Arnold, S. - Der "Aufbewahrungsort des Falschen"

Erzählillusion, ${ }^{18}$ die dem Leser die Gemachtheit und somit auch die Zufälligkeit und Willkürlichkeit der Verkettungen einzelner Handlungselemente vor Augen führt.

Solche illusionsbrechenden Verfahren kommen indes auch innerhalb des Romans zur Anwendung, so beispielsweise kurz nach dem zweiten Auftauchen des IchErzählers:

\begin{abstract}
Mit einigen harmonischen Akkorden könnte man das Buch also ausklingen lassen. Ein kurzes Landschaftspanorama vielleicht noch, ein Kameraschwenk über den gezackten Schattenriss des Kangeeri-Gebirges vor abendlicher Dämmerung, in rosa und lila Dunst getauchte Felder, Schluchten voller prupurnem Schatten, ein paar Fledermäuse, ein malerisches Maultier. Ry Cooder spielt Gitarre. Von links wandert ein Windrad ins Bild (HERRNDORF 2011: 451).
\end{abstract}

Hier wird Bezug genommen auf das Schema des Wüstenromans und dessen typische Erzählmuster. Erneut wird somit in metafiktionaler Weise die Rezeptionshaltung des Lesers diskutiert. Dieser ist gewohnt, bestimmte Erzählmuster vorzufinden und folgt bei der Lektüre des Romans den durch ein bestimmtes Genre hervorgerufenen Erwartungen. Wie ERLL/NüNNING (2005) nachweisen, sind Leser im Sinne eines Gedächtnisses der Literatur mit dem Wissen um bestimmte Gattungen ausgestattet. Dieses Wissen wenden sie bei der Konstruktion der fiktionalen Welt an. In der Besprechung des Romans in den deutschsprachigen Feuilletons spielte genau diese Einordnung eine entscheidende Rolle, und so wurde der Roman abwechselnd als Wüstenthriller, Abenteuerroman, Spionageroman, von Herrndorf selbst ironisch als „Trottelroman“"19 ${ }^{\text {gehandelt. }}{ }^{20}$ Während von einigen Kritikern die Frage nach dem Genre als zweitrangig eingestuft wurde, ${ }^{21}$ geht dieser Beitrag davon aus, dass im Rahmen des Spiels mit dem Leser und dessen Rezeptionsverhalten, das sich in der wechselnden Erzählposition und durchmischten Chronologie durch den gesamten Roman zieht, eine bewusste Vermischung von Genres vorliegt, die ihrerseits bestimmte

18 Zur Unterscheidung von Geschehens- und Erzählillusion vgl. HAUTHAL (2007: 10).

19 http://www.faz.net/aktuell/feuilleton/buecher/autoren/im-gespraech-wolfgang-herrndorf-wann-hat-estschick-gemacht-herr-herrndorf-1576165.html (31/01/2013).

20 Vgl. auch folgenden Eintrag in Herrndorfs Blog vom 25.09.2012: „Joachim hat Sand zur Hälfte gelesen und stellt die Frage, die ich mir auch schon lange gestellt habe: Was ist denn das nun eigentlich? Der Verlag hat es mal Richtung Thriller gelabelt, aber es ist ein weites Feld zwischen Unterhaltungs-, Schund- und Gesellschaftsroman, von Thor Kunkel bereits mäßig erfolgreich beackert." http://www.wolfgang-herrndorf.de/2011/09/zwanzig/ (31/01/2013).

21 „Klar ist auch, dass sich das Genre nicht eindeutig bestimmen lässt, und ebenso klar, dass deshalb auch nicht der schmächtigste Reissack in China umkippt“ (MAAR 2012: 334). 
Erwartungen beim Rezipienten auslösen. Dies wird an vorliegender Stelle selbstreflexiv thematisiert. Der Leser wird sich damit der implizit gehegten Erwartungen bewusst und kann darauf reflektieren. Ein Verfahren, das von Birgit NEUMANN (2006: 93) als illokutionär bezeichnet wird: „Indem sie kulturelle Standards kritisch perspektiviert und mit Erwartungen spielt, entfaltet Literatur eine illokutionäre Kraft: Sie veranlasst ihre Rezipienten zu einer veränderten Wahrnehmung kulturell etablierten Wissens und kann damit in der Kultur als formative, weltverändernde Kraft wirksam werden.“ An dieser Stelle wird sich der Leser seines vorgeformten Rezeptionsverhaltens bewusst, was gleichzeitig als metafiktionaler Kommentar zum gesamten Roman und dessen ständigen Brüchen mit vorgeformten Erzählmustern und Erwartungen fungiert.

Die Gemachtheit der Geschichte wird nochmals deutlicher, wenn es im Anschluss heißt „Man könnte allerdings auch [...] noch einen Blick zurück auf eine nicht ganz unwesentliche Figur dieser Geschichte werfen [...]“ (HERRNDORF 2011: 451/52) und somit verschiedene Konstruktionsmöglichkeiten der Geschichte gleichzeitig nebeneinander stehen. Das Ende der Geschichte, das zunächst vermeintlich auf ein Happy End hinauszulaufen scheint, indem der Protagonist durch unwahrscheinliche Zufälle doch noch die Befreiung aus der Höhle schafft, was sich der Leser jedoch ,allein in seiner Phantasie“ (HERRNDORF 2011: 453) vorstellen soll, bricht dementsprechend erneut mit den Erwartungen. In Erwartung eines glücklichen Endes wird Carl, ganz von Zufall und Willkür motiviert, eine Kugel in den Kopf geschossen. Der gewalttätige Impetus eines Teils der Marseillaise, die dem letzten Kapitel als Motto vorangestellt ist, wird sowohl im zufälligen Tod Carls als auch im sinnlosen Tod des kleinen Mädchens als Endton des Romans aufgegriffen, der damit zu Recht als das Gegenstück seines Vorgängerromans Tschick gelten kann (vgl. AKRAP 2012). Das Motiv des Todes, das zuvor bereits mit dem Motiv der Dunkelheit verbunden war, wird dabei ebenfalls mit dem Zufall kombiniert und entfaltet sich, obwohl es unterschwellig während des ganzen Romans präsent war, erst in dem Moment, in dem es vom Leser am wenigsten erwartet wird. Dass Herrndorf mit genreabhängigen Erwartungen spielt, zeigt auch sein Kommentar zur Aufnahme des Elixiermotivs in beide Romane (vgl. HÜNNIGER 2012). In Tschick wird das Elixier vom Alten mit der Flinte an die Jugendlichen weitergegeben, die es dann aus dem Fenster werfen. In Sand verliert der Protagonist es an die Slumkinder. Nach diesem Motiv befragt, äußert Herrendorf im Gespräch mit Kathrin Passig: 
[...] oder an der Szene mit dem Elixier. Das bin ich jetzt auch schon häufiger gefragt worden: Was das für ein Elixier ist, das der Alte mit der Flinte den beiden da aufdrängt? Aber das weiß ich ja auch nicht. Das war nur, weil mich beim Schreiben jemand auf die „Heldenreise“ aufmerksam machte, ein Schema, nach dem angeblich fast jeder Hollywood-Film funktioniert. Da müssen die Protagonisten unter anderem immer ein solches Elixier finden. Habe ich natürlich gleich eingebaut. (PASSIG 2011).

Der Unterschied zum traditionellen Plot der Heldenreise besteht darin, dass das gefundende Elixier zwar die Rettung verspricht - so ist Carl davon überzeugt, in der Mine die Lösung des Rätsels gefunden zu haben und seinem Gedächtnis so auf die Sprünge zu helfen -, es aber durch einen Zufall wieder verlorengeht und in der Bedeutungslosigkeit verschwindet. Ähnlich wird mit dem kurzzeitig greifbar nahen Happy End verfahren, das zuvor jedoch schon durch den metafiktionalen Verweis auf verschiedene Möglichkeiten für ein Ende dekonstruiert wurde.

Während sowohl die erzählerischen Brüche als auch das Spiel mit den Gattungskonventionen als metaisierende Verfahren gelten können (vgl. HAUTHAL: 8), sind damit noch nicht ihre Funktionspotentiale geklärt. Die Erzählperspektive, die stellenweise auktorial und allwissend wirkt (vgl. z.B. HERRNDORF 2011: 82), an zwei Stellen von einem rahmenden Ich-Erzähler durchbrochen wird, an anderen Stellen als personal oder als geradezu cinematisches Erzählen (HERRNDORF 2011: 323, 365, 452) zu kennzeichnen ist, trägt wesentlich zur Desorientierung des Lesers bei. Durch die Vielzahl von Erzählpositionen, die Rahmenhandlung mit Fiktionsbruch sowie das Spiel mit den Plotmustern werden die zentralen Themen des Romans - Unordnung, Sinnlosigkeit und Zufall - auch auf der formalen Ebene figuriert. Auf der Ebene der histoire wird der Roman durch zahlreiche semantische Spiele zum „Aufbewahrungsort des Falschen“.

\section{Homonyme und Homophone: die Mi(e)ne}

Die semantischen und phonetischen Doppeldeutigkeiten des Romans reihen sich größtenteils um das Wort Mine (auch in der Schreibweise Miene), das zum ersten Mal völlig unvermittelt bei Helens Ankunft von einem Taxifahrer aufgegriffen wird: 
Arnold, S. - Der "Aufbewahrungsort des Falschen"

Der Taxifahrer hatte nur einen linken Arm und schaltet die Gänge, indem er den Oberkörper herumdrehte, während er mit den Knien das Steuer festhielt. «Mine», sagte er und wedelte mit der kahlen rechten Schulter. Es war sein einziger Beitrag zur Konversation. (HERRNDORF 2011: 46).

Obgleich dieses Wort und seine diversen semantischen Konzepte Triebfeder für einen großen Teil der Handlung sind, kann ihm weder an dieser noch an anderer Stelle eine klar definierte Bedeutung für den Fortgang der Handlung zugeordnet werden. Hier kommt das Wort wie aus dem Nichts und kann bestenfalls als vorausdeutend auf das folgende Geschehen angesehen werden, an anderen Stellen generiert es vermeintlich Bedeutung und Kausalzusammenhänge, so beispielsweise, wenn Carl glaubt, endlich das Geheimnis der Mine entschlüsselt zu haben und somit die Handlungsfäden verknüpfen zu können - indes Kausalzusammenhänge, deren logische Verknüpfungen im nächsten Moment wieder zerstört werden. ${ }^{22}$

Erneut aufgegriffen wird das Konzept der Mine, wenn der an Amnesie leidende Protagonist sich an das Gespräch von vier Männern beim Erwachen in einer Scheune zu erinnern versucht. Demnach gibt es folgende Möglichkeiten für das Gespräch zwischen ihnen: „Wenn er die Schiene... die Maschine... Christine jetzt verhört. Vier Männer in weißen Dschellabhas, ein Bastkoffer, ein Jeep“ (HERRNDORF 2011: 93). Der Logik des Knapp daneben folgend, werden hier ähnlich klingende Wörter auf ihre Sinnhaftigkeit abgetastet. Nur durch Helens Hinweis geht Carl überhaupt davon aus, dass die Mine das entscheidende Element sein könnte: „Wenn er Pauline informiert, wenn er die Bienen exportiert, wenn die Maschine funktioniert... ich weiß es nicht.“ (HERRNDORF 2011: 135), fährt Carl im Gespräch mit ihr fort. „Wenn er die Mine jetzt zerstört“ (HERRNDORF 2011: 135), korrigiert Helen und legt damit die Basis für die Reflexionen um die Mine. Dass es sich hierbei indes genausogut um einen Fehlschluss handeln kann, wird im Roman nur indirekt thematisiert, indem von einem Mann die Rede ist, „der weder willentlich noch zufällig unter die Räder des Schicksals geriet, sondern einzig und allein durch eine falsche logische Schlussfolgerung; durch den Glauben an die Unschuld eines Schuldigen“" (HERRNDORF 2011: 452). Diese falsche logische Schlussfolgerung kann gleichermaßen auf die falsche Interpretation einer vagen

22 „Wie schon zuvor hatte er das Gefühl, dass mit Nachdenken etwas zu holen sei, aber immer, wenn er die Fäden zu verknüpfen suchte, verhedderten sie sich sofort in seinen Händen, und dann fuhr ein heftiger Windstoß durch seine Überlegungen, der nicht nur alle Verknüpfungen löste, sondern auch die Fäden selbst in luftige Fernen davonwehte" (HERRNDORF 2011: 352). 
Erinnerung bezogen werden, die phonetisch ähnlich klingende Wortfetzen bereithält und mit der Interpretation des Homonyms Mine und seines Homophons Miene weitere Möglichkeiten für logisch falsche Verknüpfungen in sich birgt. Der Roman figuriert damit auch die Fehlschaltungen eines beschädigten Gehirns. In Neurobiologie und Psychologie existieren mehrere Theorien dazu, dass die Bedeutungen von Homophonen im Gehirn in ähnlichen Netzen gespeichert sind. ${ }^{23}$ In Carls beschädigtem Gehirn wäre demnach ein bestimmter, nicht näher eingrenzbarer Bereich aktiv, in dem sich im Sinne eines großen Speichers mit einzelnen Subkarteien Homophone und Homonyme von Mine befinden. Auf die Vorstellung des Gehirns als Sammelort von Karteikarten (vgl. SPITZER 1996) rekurriert der Roman selbst, wenn „Worte wie Dachboden, Bretterwand, Amnesie, Flaschenzug, Titrierkolben und Sandhaufen aufklappen wie Memorykarten“ (HERRNDORF 2011: 92). Herrndorfs Blog Arbeit und Struktur ist voll mit Reflexionen zu phonetischen und semantischen Ähnlichkeiten und ihren Verknüpfungsmöglichkeiten:

\subsection{5:35}

Meine Gedanken werden zunehmend laut in mir, in allen Geräuschen schwimmen Silben und Sätze. Der gleichmäßige Atem C.s schwillt an wie Meeresbrandung und treibt mich auf, zwischen sich selbst sagenden Sätzen und Sprachverlust, zwischen innerer Stimme und Epilepsie ist kaum noch ein Unterschied. ${ }^{24}$

Im Roman werden in der Folge die Mine als Bergwerk, als Bleistiftsmine, als Sprengstoff, als Münze und das Homophon Miene (Gesicht) differenziert: „Die Miene im Gesicht, das Bergwerk. Das Sprengding und das in den Bleistiften“ (HERRNDORF 2011: 162, vgl. auch die Recherche im Brockhaus, 216). Auffallend oft wird das Wort Mine dabei durch „Dings“ (HERRNDORF 2011: 398) ersetzt (vor allem während der Folter am Ende des Romans), als sei semantisch ohnehin klar, was gemeint ist, und die phonetische Auskleidung nur ein Zusatz. Hinzu kommen schließlich noch das Restaurant Zur Goldmine (HERRNDORF 2011: 174) und der Traum von Helens Miene (HERRNDORF 2011: 174/175). Die davon verschiedene Schreibweise des Homophons wird parallel dazu im Flyer des Psychiaters in Helens Briefkasten aufgegriffen: „Termiene mit ie“ (HERRNDORF 2011: 186).

23 Vgl. als Grundlage hierfür das Logogen-Modell von MORTON (1969) und das Cohorten-Modell von MARSLEN-WILSON/WELSH 1978.

24 http://www.wolfgang-herrndorf.de/2012/10/dreiunddreissig/ (31/01/2013). 
Immer wieder wird im Roman darauf hingewiesen, dass es sich bei Carls Gedächtnisschädigung um eine retrograde Amnesie handelt, bei der lediglich das autobiographische bzw. episodische Gedächtnis beschädigt ist, während das semantische Gedächtnis bestens funktioniert (vgl. etwa: „Er fragte sich, woher er überhaupt wusste, dass es so etwas wie Gedächtnisverlust gab? In welchem Leben hatte er das gelernt?“" (HERRNDORF 2011: 114). Indes wird, wie bereits gezeigt, dieser Gedächtnisteil durch eine Vielzahl von semantischen Spielereien ebenfalls auf die Probe gestellt. Dies geschieht beispielsweise, wenn der Psychiater Dr. Cockcroft Carl nach der Dreyfuß-Affäre befragt. Indes ist damit keinesfalls die gemeinhein als Dreyfuß-Affäre bekannte Verurteilung des jüdischen Hauptmanns Alfred Dreyfuß im Paris des Jahres 1894 gemeint. Wie der Psychiater im Anschluss erklärt, geht es in seinem Beispiel um den am MIT tätigen US-amerikanischen Philosophen Hubert Dreyfus, der als Kritiker der künstlichen Intelligenz bekannt wurde und ,als erster Mensch, der dümmer war als ein paar Kupferdrähte“ (HERRNDORF 2011: 199) in die Geschichte einging. Zunächst nimmt Cockcroft hier auf ein Konzept Bezug, das gemeinhin mit einem anderen Inhalt, nämlich der Affäre um den elsässischen Offizier Ende des 19. Jahrhunderts, verbunden wird, dann aber im Sinne des „Aufbewahrungsort des Falschen“ mit einem namensähnlichen, semantisch jedoch völlig andersartigen Konzept verbunden wird. Indes wird der Zweck dieser Anekdote im Therapiegespräch nicht deutlich. Als Schlussfolgerung konstatiert Dr. Cockcroft lediglich:

\footnotetext{
So oder so ähnlich muss man sich auch das Gehirn vorstellen. Man denkt sich das eigene Organ notgedrungen als etwas höchst Kompliziertes und Fragiles, weil man seine Äußerungen - ob zu Recht oder zu Unrecht - als kompliziert und fragil empfindet. Aber auf der rein physischen Ebene gibt es keine Entsprechung zu dieser Empfindlichkeit, und man erzielt gute Resultate mit Schraubenzieher und Kneifzange (HERRNDORF 2011: 198).
}

Cockcroft propagiert hier eine Sichtweise des Gehirns, die weder mit den realbiologischen Erkenntnissen der Neurowissenschaftler (vgl. MARKOWITSCH 2000), noch mit der auf der fiktionalen Ebene postulierten Sicht des Gehirns in Einklang zu bringen ist. Im Roman sind es winzige Fehlschaltungen im Gehirn, die zu Fehlinterpretationen und Verkettungen zufälliger unglücklicher Umstände führen und gerade darin schließlich zum Tod mehrerer Figuren. Ein ähnlicher Fehlschluss zwischen phonetischer und semantischer Ähnlichkeit lässt sich beim Konzert der Hippie-Band 
Arnold, S. - Der "Aufbewahrungsort des Falschen"

Marshall Mellow aufzeigen, wenn der gleichnamige Sänger mit einem Mal über das Marshmallow-Experiment singt - ein Experiment mit Realitätsreferenz, bei dem Selbstund Impulskontrolle bei Kindern getestet wurden. Der Zusammenhang scheint auch hier nur phonetisch zu sein, wenn auch dies aufgrund der personalen Erzählperspektive nicht eindeutig $\mathrm{zu}$ klären ist: „einerseits schienen die Worte dunkel und unzusammenhängend, andererseits brachten sie die ersten Hippies im Parkett bereits zum Ausrasten“ (HERRNDORF 2011: 355). Der Roman nimmt damit implizit mehrere Theorien zu semantischer und phonetischer Repräsentation auf, die Entsprechungen in Psychologie, Neurowissenschaften und Linguistik haben und im Roman mit logischen Fehlschlüssen und falschen Fährten verbunden sind.

Als Carl auf der Suche nach dem Geheimnis der Mine den Herumtreiber Risa in einer Bar nach mehreren Bedeutungen des Homonyms Mine fragt, wird dieser misstrauisch:

Du willst wissen, ob es hier irgendein Bergwerk gibt? Und was willst du dann damit? Willst du mit irgendeiner Mine, die du nicht kaufen willst, irgendein Bergwerk in die Luft jagen, von dem du nicht weißt, ob es existiert?“ „Da ist kein Zusammenhang, ich weiß. Das eine hat mit dem anderen nichts zu tun.“ „Außer dass beides Mine heißt.“ „Ja. Aber das ist Zufall.“” „Das ist Zufall? Was ist Zufall?“ „Seit wann ist das Zufall, wie was heißt? Mine und Mine. Du meinst, das ist Zufall? So scheißintellektuell bist du denn auch wieder nicht, was? (HERRNDORF 2011: 236).

Obgleich Carl während des gesamten Romans von diversen Seiten (sowohl als Polidorio als auch als Carl) mangelnde Intelligenz bescheinigt wird, ${ }^{25}$ nimmt er hier implizit auf die in der Semiotik allgemein akzeptierte Theorie des Linguisten Ferdinand de Saussure von der Arbitrarität des Zeichens Bezug: ,Jedes Zeichen hat einen Wert nur durch seine Opposition zu den anderen Zeichen des Systems.“ (apud MAHMOUdiAN 1973: 258). Zwar hat die folgende Theorie (Bergleute hatten im Mittelalter Festungen untergraben, die dann eingestürzt sind und somit den Zusammenhang zwischen Sprengstoff und Bergwerken erklärt) durchaus Hand und Fuß, auf die weiteren Bedeutungen des Homonyms Mine trifft dies aber nicht $\mathrm{zu}$ - in diesem Fall handelt es sich streng

25 Helen sagt am Ende über ihre Hoffnung, ihr Vorhaben vor Carl nicht auffliegen zu lassen: „lass ihn bitte exakt so dämlich sein, wie er aussieht" (HERRNDORF 2011: 424). Noch in seiner Identität als Polidorio führt Carl einen Intelligenztest mit dem durchschnittlichen Resultat 102 durch (HERRNDORF 2011: 9-11). Indes liefert der Roman auch Hinweise darauf, dass Helens Einschätzung eine falsche ist; der Intelligenztest wurde zudem unter dem Einfluss von Alkohol durchgeführt und von einem Stromausfall unterbrochen. 
Arnold, S. - Der "Aufbewahrungsort des Falschen"

genommen um eine Polysemie, denn beide Bedeutungen stammen etymologisch von einem Lexem. Zudem können sowohl Homonyme als auch Polyseme nicht ohne Weiteres auf andere Sprachen übertragen werden. Das im Roman herrschende Sprachengewirr zwischen Französisch, Arabisch, Englisch und Deutsch lässt keine Schlüsse auf Übersetzungsprobleme zu, zumal im auf Deutsch geschriebenen Roman noch Spiele mit den verschiedenen Sprachen eingebaut werden - so wenn Helen Carl den wahren Zweck ihrer Mission offenbart: „Herrlichkoffer, das ist deutsch und heißt so viel wie Herrlichkoffer“ (HERRNDORF 2011: 421).

Einerseits steht die Thematik des Gedächtnisverlusts eng mit der Identitätssuche des Protagonisten in Zusammenhang, ein Thema, das Herrndorf bereits in seiner preisgekrönten Kurzgeschichte Diesseits des Van-Allen-Gürtels ${ }^{26}$ und im Vorgängerroman Tschick aufgegriffen hatte. Andererseits ist die Simulation eines beschädigten Gehirns und seiner logischen Fehlschaltungen auch auf die Handlung des Romans zu beziehen, die durch Zufälle und falsche logische Schlussfolgerungen bestimmt ist. Sie hängt in dieser Sichtweise auch wesentlich mit dem Wüstenmotiv als Ort des Absurden und Unbestimmbaren sowie mit den formalen Kriterien um Plotstruktur, zeitliche Anordnung und Erzählposition, die das Rezeptionsverhalten bestimmen, zusammen.

\section{Schluss}

Wolfgang Herrndorfs Roman Sand ist sowohl auf der formal-ästhetischen als auch auf der intradiegetischen Ebene durch Fehler, Missverständnisse und Bedeutungsverschiebungen gekennzeichnet. Ausgehend von der Wüstenkonnotation, die dem Roman bereits durch das Umschlagbild zu Grunde liegt und innerhalb des Romans in verschiedenen Traditionslinien weitergeführt wird, lässt sich eine Logik der Zufälligkeit und der Missverständnisse nachzeichnen. Die Wüste ist dabei Ort des Transitorischen, des Unergründlichen und Unabschließbaren, aber auch der Ort des Bösen. Sie kann in unterschiedlichen Interpretationen entweder Selbstfindung oder

26 Dort versucht sich der Ich-Erzähler unter Alkoholeinfluss vergeblich an seine Kindheit und seine damaligen Vorstellungen von der Welt zu erinnern. Die Geschichte gewann 2004 den KelagPublikumspreis beim Ingeborg-Bachmann-Wettbewerb. 
Arnold, S. - Der "Aufbewahrungsort des Falschen"

Selbstverlust bedeuten. Für den Protagonisten wird sie jedoch, der Philosophie des Absurden folgend, zum Ort des Sprach- und Selbstverlusts, der bereits durch die von der Wüstensonne ausgelösten Sprachstörungen Lundgrens vorweggenommen wird. Das von der Wüstensonne gestörte Gleichgewicht lässt sich auch auf die Ebene der Rezeption übertragen. Eine nicht immer klar $\mathrm{zu}$ dechiffrierende Zeitstruktur, unzuverlässige Reflektorfiguren sowie eine Vielzahl von Erzählpositionen übertragen die Unsicherheit bei der Entschlüsselung der Geschehnisse auf den Leser. Zudem wird mit gängigen Erzählmustern und Leseerwartungen gespielt, so beispielsweise mit dem Genre des Wüstenromans oder mit der Erwartung eines Happy Ends, das indes gleichzeitig metafiktional dekonstruiert wird. Inhaltlich lässt sich dies anhand des Spiels mit semantischen Mehrdeutigkeiten nachzeichnen - zum einem als Spiel mit dem Homonym Mine und seines Homophons Miene, zum anderen mit semantischen Konzepten und ihren Assoziationen, wie beispielsweise der Dreyfus-Affäre oder des Marshmallow-Experiments. Der Roman spielt somit auf mehreren Ebenen die Logik des Knapp daneben, Missverständnisse und Fehldeutungen durch, gestaltet damit eine fiktionale Welt des Absurden und wird zum „Aufbewahrungsort des Falschen“, wie Herrndorf in seinem Blog Arbeit und Struktur konstatiert:

Lese meine eigenen Dialoge und stelle fest, daß ich das Mißverständnis für das Wesen der Kommunikation halte. Es werden Fehler gemacht, und die Fehler führen zu allem. Man könnte auch Zufälle sagen, aber das Wort Fehler ist mir lieber. Ich halte den Roman für den Aufbewahrungsort des Falschen. ${ }^{27}$

\section{Literaturverzeichnis}

AKRAP, Doris. „Das muss man erstmal hinkriegen.“ Interview mit Herrndorfs Freund Robert Koall. In: taz, http://www.taz.de/1/archiv/digitaz/artikel/?ressort=tz\&dig=2012/03/15/a0191\&cHash= 158f3a11e0 $(31 / 01 / 2013)$.

BubER, Martin. Ich und Du. In: Ders.: Werke. Erster Bd. Schriften zur Philosophie. München: Kösel, 1962, 77-170.

CAmus, Albert. Der Fremde. (Uli Aumüller, Übers.). Reinbek bei Hamburg: Rowohlt, 1994.

Camus, Albert. Der Mythos des Sisyphos. (Vincent von Wroblewsky, Übers.). Reinbek bei Hamburg: Rowohlt, 1999.

27 http://www.wolfgang-herrndorf.de/2011/09/zwanzig/ (31/01/2013). 
Arnold, S. - Der "Aufbewahrungsort des Falschen"

EDER, Jens. Die Figur im Film. Grundlagen der Figurenanalyse. Marburg: Schüren, 2008.

ERLL, Astrid / NÜNNING, Ansgar. Literaturwissenschaftliche Konzepte von Gedächtnis: Ein einführender Überblick. In: ERLL, Astrid / NÜNNING, Ansgar (Hg.). Gedächtniskonzepte der Literaturwissenschaft. Theoretische Grundlegung und Anwendungsperspektiven. Berlin: de Gruyter, 2005, 1-9.

GENETTE, Gérard. Die Erzählung. 2. Aufl. (Andreas Knop, Übers.). München: Fink, 1998.

GOETSCH, Paul. Identitätskonstruktionen in Robinson Crusoe. Zur verführerischen Macht eines autobiographischen Erzählmusters. In: NEUMANN, Michael (Hg.): Erzählte Identitäten. München: Fink, 2000, 90-105.

Hauthal, J./ NADJ, J./ NÜNNING, A. et al. (Hg.). Metaisierung in Literatur und anderen Medien: Begriffserklärungen, Typologien, Funktionspotentiale und Forschungsdesiderate. Berlin: de Gruyter, 2007, 1-21.

HERRNDORF, Wolfgang. Sand. Berlin: Rowohlt, 2011.

HERRNDORF, Wolfgang. Tschick. 12. Aufl. Berlin: Rowohlt, 2011.

HERRNDORF, Wolfgang. Arbeit und Struktur: http://www.wolfgangherrndorf.de/2011/09/zwanzig/ (31/01/2013).

HÜNNIGER, Andrea Hanna. Die Wüste ist ein sinnloser Ort. In: Die Zeit, 22.11.2011: http://www.zeit.de/2011/47/L-B-Herrndorf (31/01/2013).

KAiser, Christine. Homonymie. In: Burdorf, D./ FAsBender, C./ Moennighoff, B. (Hg.). Metzler Lexikon Literatur. 3. Aufl. Stuttgart; Weimar: Metzler: 2007, 326.

Kaiser, Christine. Phraseologie. In: Burdorf, D./ FAsbender, C./ Moennighoff, B. (Hg.). Metzler Lexikon Literatur. 3. Aufl. Stuttgart; Weimar: Metzler: 2007, 585.

KANDEL, Eric. Auf der Suche nach dem Gedächtnis. 3. Aufl. (Hainer Kober: Übers.). München: Panthenon, 2009.

KIRCHHOFF, Bodo. Der Sandmann. Frankfurt/Main: Suhrkamp, 1992.

LATZ, Michael. NDR 2: Buchbesprechung (27.05.2012). Wolfgang Herrndorf: Sand: http://www.ndr.de/ndr2/programm/sendungen/buchtipp/buchtipp799.html (31/01/2013).

LindEMANN, U./ SCHMITZ-EMANS, M. (Hg.). Was ist eine Wüste? Interdisziplinäre Annäherungen an einen interkulturellen Topos. Würzburg: Königshausen \& Neumann, 2000.

MAAR, Michael. „Er hat's mir gestanden.“ Überlegungen zu Wolfgang Herrndorfs Sand. In: Merkur 4, 2012, 333-340.

Mahmoudian, Morteza. Zeichen. In: Martinet, André (Hg.). Linguistik. Ein Handbuch. Stuttgart: Metzler, 1973, 258.

MARKOWITSCH, Hans J. Die Anfälligkeit autobiographischer Erinnerung gegenüber Streß. Eine neuropsychologische Perspektive. In: NeumanN, Michael (Hg.). Erzählte Identitäten. München: Fink, 2000, 215-229.

MARSLEN-WiLson, W./ Welsh, A.: Processing Interactions and Lexical Access during Word Recognition in Continuous Speech. In: Cognitive Psychology 10 (1), 1978, 29-63.

MORITZ, Rainer. Wenn kein Sandkorn auf dem anderen bleibt. In: Deutschlandradio Kultur: Radiofeuilleton, 08.12.2011: http://www.dradio.de/dkultur/sendungen/kritik/1622678/ (31/01/2013).

Neumann, Birgit. Performanz und Literatur: Vorschläge zur Konzeptualisierung der TextKontext-Relationen. In: GYMNICH, Marion (Hg.). Kulturelles Wissen und Intertextualität. Theoriekonzeptionen und Fallstudien zur Kontextualisierung von Literatur. Trier: WVT, 2006, 87-106.

NÜNNING, Ansgar. Unreliable Narration zur Einführung: Grundzüge einer kognitivnarratologischen Theorie und Analyse unglaubwürdigen Erzählens. In: NÜNNING, 
Arnold, S. - Der "Aufbewahrungsort des Falschen"

Ansgar (Hg.). Unreliable Narration. Studien zur Theorie und Praxis unglaubwürdigen Erzählens in der englischsprachigen Erzählliteratur. Trier: WVT, 1998, 3-39.

PASSIG, Kathrin. Wann hat es „Tschick“ gemacht, Herr Herrndorf? Kathrin Passig im Gespräch mit Wolfgang Herrndorf. In: FAZ, 31.01.2011: http://www.faz.net/aktuell/feuilleton/buecher/autoren/im-gespraech-wolfgangherrndorf-wann-hat-es-tschick-gemacht-herr-herrndorf-1576165.html (31/01/2013).

SCHNEIDER, RALF. Grundriß zur kognitiven Theorie der Figurenrezeption am Beispiel des viktorianischen Romans. Tübingen: Stauffenburg, 2000.

SPITZER, Manfred. Geist im Netz. Modelle für Lernen, Denken und Handeln. Heidelberg;

Berlin; Oxford: Spektrum Akademischer Verlag, 1996, Kap. 10.

WeLZER, Harald: Das kommunikative Gedächtnis. München: Beck, 2002.

Recebido em 01/03/2013

Aprovado em 05/04/2013 